\title{
BENDS IN NON-RADIATIVE DIELECTRIC GUIDE
}

\author{
P. BHUSHAN MITAL, Fellow IETE \\ Department of Electronics \& Communication Engg., CR State College of Engineering, \\ Murthal (Sonepat), India 131039
}

(Received April 23, 1994; in final form May 11, 1994)

Curved bends in NRD waveguides have been developed. Measured insertion losses of $90^{\circ}$ and $180^{\circ}$ bends in an NRD guide are reported. Both the bends have insertion losses very near a reference straight guide in the frequency range from $33.5-36.5 \mathrm{GHZ}$.

\section{INTRODUCTION}

At millimeter wave frequencies, various dielectric integrated guides are available as transmission media vis-a-vis an image guide, insular image guide, trapped image guide, and non-radiative dielectric waveguide. A non-radiative dielectric guide (NRD) is similar to an $\mathrm{H}$ guide but differs in principle of operation [1]. In an $\mathrm{H}$ guide, the two paralle conducting planes are more than half a wavelength apart, whereas, in an HRD guide, the separation of two planes is kept less than half a wavelength. The electromagnetic field with E-vector parallel to planes cannot propagate in the air medium between the planes because of their cut-off property as shown in Fig. 1. But surface waves are able to propagate freely along with dielectric strip, whether it be straight or curved. Radiated waves, if any, decay outside the strip and, hence, bends and junctions can easily be incorporated into complicated integrated circuits $[2,3]$.

Normal dielectric waveguides have formidable radiation losses at curved sections. The bending losses consists of (a) radiation loss and (b) reflection loss at the bends. Field profile in a curved dielectric guide is not symmetric about the central axis of guide. Field maxima is shifted outwardly and a significant portion of energy exists on the outer side of the curved guide. Thus, the energy remains loosely bound at the curved section and radiates. On the inner side, fields decay radially much faster than that in a straight guide. Therefore, a large radius of curvature is a necessity to approximate the fields to a straight guide.

Since separation of two conducting parallel planes is less than half of the free space wavelength, the radiation mode remains completely cut off in an NRD guide. Yoneyama has shown mathematically that the effective dielectric constant for nonradiative dielectric core region and therefore, electromagnetic tunneling [3] never occurs. Hence, there is no radiation loss at all at the bends. Bending losses in nonradiative dielectric guides are due to reflection alone at the transitions between straight guide and curved guide $[4,5]$. 


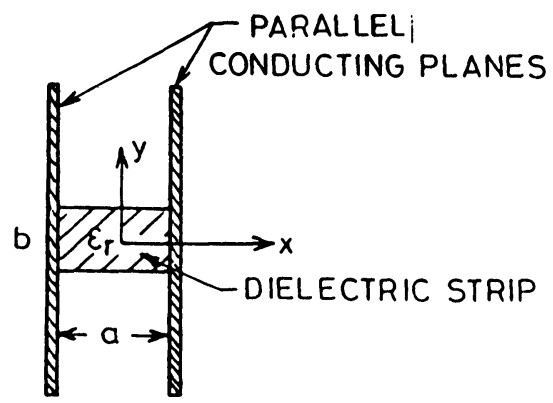

(A)

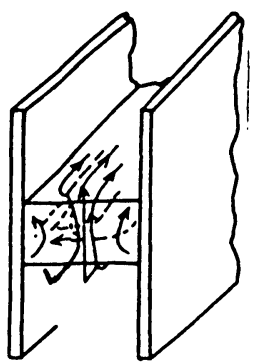

(B)

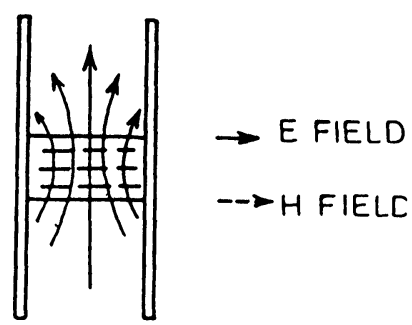

(C)

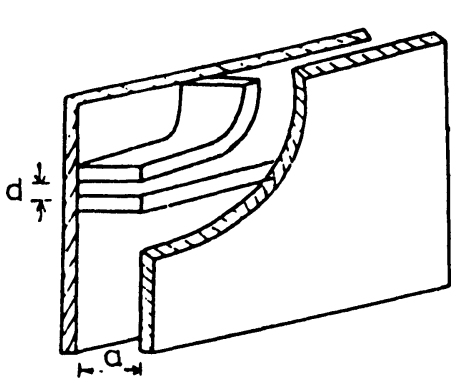

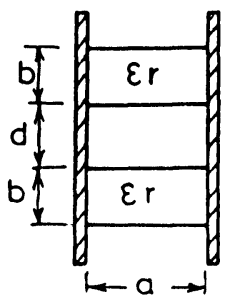

EDGE COUPLED COMPONENT IN NRD

(D)

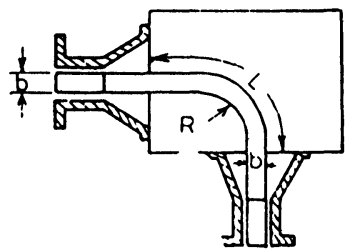

(G)

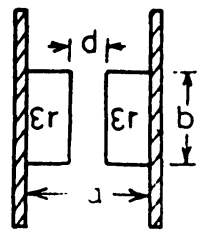

BROAD SIDE COUPLED NRD

(F)

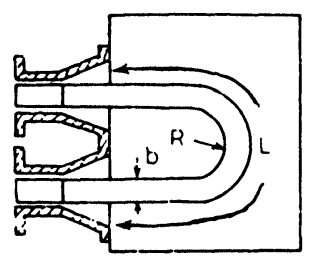

(H)

FIGURE 1

\section{FABRICATION OF BENDS}

In a non-radiative dielectric guide, the separation of two parallel planes has to be kept constant and below a certain value in order to ensure that there is no radiation. In order to study the effect of bends, etc, the dielectric strip has to be in the $E$ 


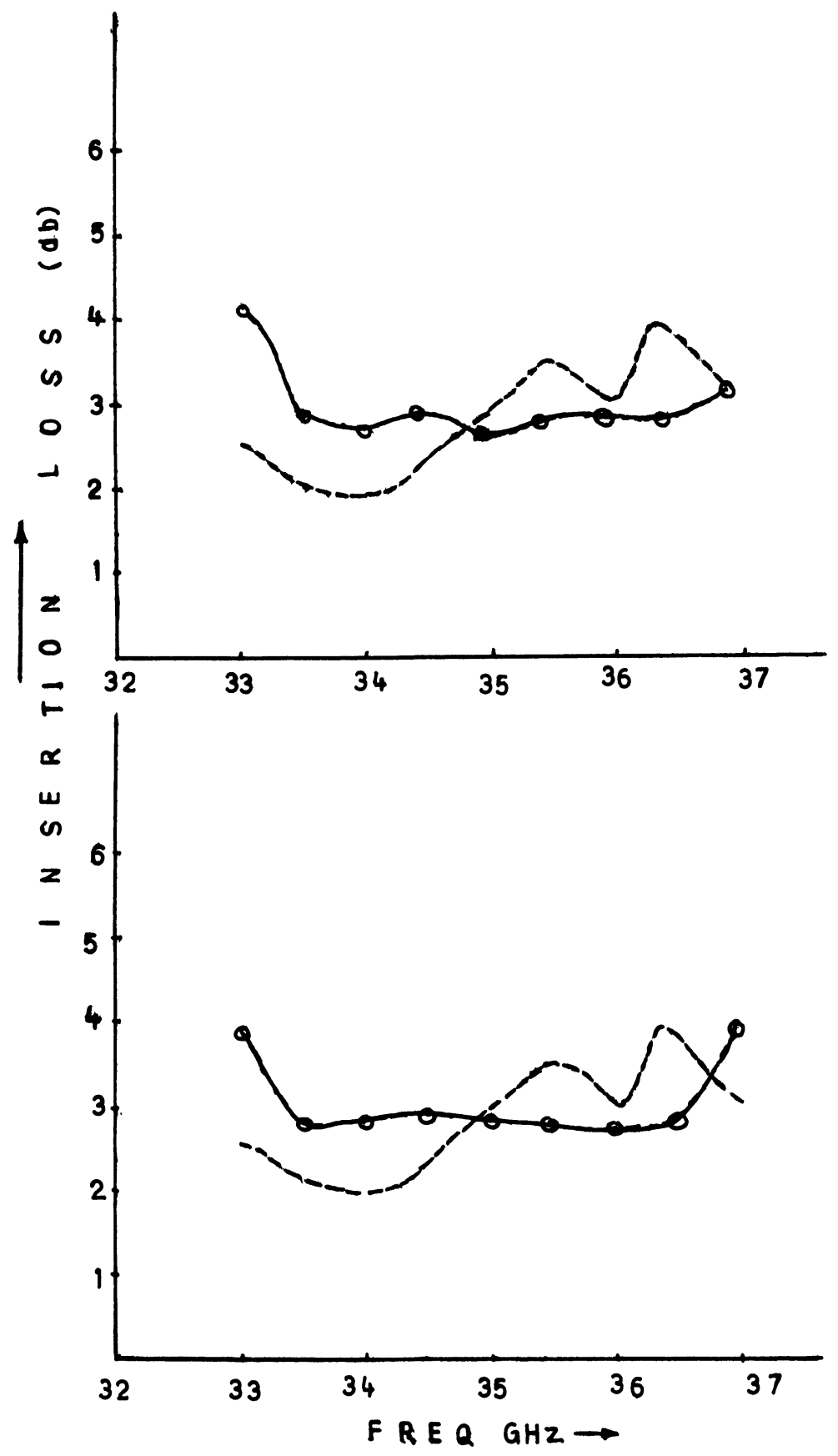

FIGURE 2 
TABLE I

Insertion losses of $90^{\circ}$ and $180^{\circ}$ bends in NRD guide.

\begin{tabular}{llll}
\hline Sr. No. & Freq. GHZ & $90^{\circ}$ bend & $180^{\circ}$ bend \\
\hline 1 & 33.0 & 4.2 & 3.8 \\
2 & 33.5 & 2.8 & 2.7 \\
3 & 34.0 & 2.7 & 2.6 \\
4 & 34.5 & 2.9 & 2.9 \\
5 & 35.0 & 2.6 & 2.8 \\
6 & 35.5 & 2.7 & 2.7 \\
7 & 36.0 & 2.8 & 2.6 \\
8 & 36.5 & 2.7 & 2.8 \\
9 & 37.0 & 3.2 & 4.0 \\
\hline
\end{tabular}

plane only, i.e., width 'a' of dielectric strip remains unaltered. The $90^{\circ}$ and $180^{\circ}$ bends with radii of curvature $\mathrm{R}=2.50 \mathrm{~cm}$ has been chosen for experiments. The ends of the dielectric strip had $1.50 \mathrm{~cm}$ long $\mathrm{H}$-plane taper to fit the transition portion.

\section{MEASUREMENTS}

Insertion loss of curved NRD guide bends of $90^{\circ}$ and $180^{\circ}$ with radius of curvature as $2.5 \mathrm{~cm}$ are given in table $I$ and plotted in fig. 2 . The insertion loss of a straight NRD guide is also plotted for comparison. It is found that insertion loss of an NRD guide tallies with the insertion loss of a straight guide.

\section{CONCLUSION}

Curved section of $90^{\circ}$ and $180^{\circ}$ with radii of curvature as $2.5 \mathrm{~cm}$ were fabricated from polystyrene dielectric. The insertion loss of the bends tallies with that of a straight guide; thus, the bends are approximated well to a straight guide.

\section{REFERENCES}

1. T. Yoneyama and S. Nishida; "Non radiation dielectric waveguide for millimeter wave integrated circuits," IEEE Trans MTT-29, pp 1188-1191, Nov. 1981.

2. T. Yoneyasma, N. Tozawa and S. Nishida; "Coupling characteristics of non-radiative dielectric guides," IEEE Trans MTT-31, pp 648-654, Aug. 83.

3. T. Trinh and R. Mitra: "Field profile in a single mode curved dielectric waveguide of rectangular cross-section," IEEE Trans MTT-29; pp 1315-1318, Dec. 81.

4. T. Yoneyama, M. Yamaguchi and S. Nishida; "Bends in NRD guides," IEEE Trans MTT-30, pp 2146-2149, Dec. 82.

5. A.W. Snyder and J.D. Love; "Reflection at curved dielectric interface-electromagnetic tunneling," IEEE Trans MTT-23, pp 134-141, Jan. 1975. 

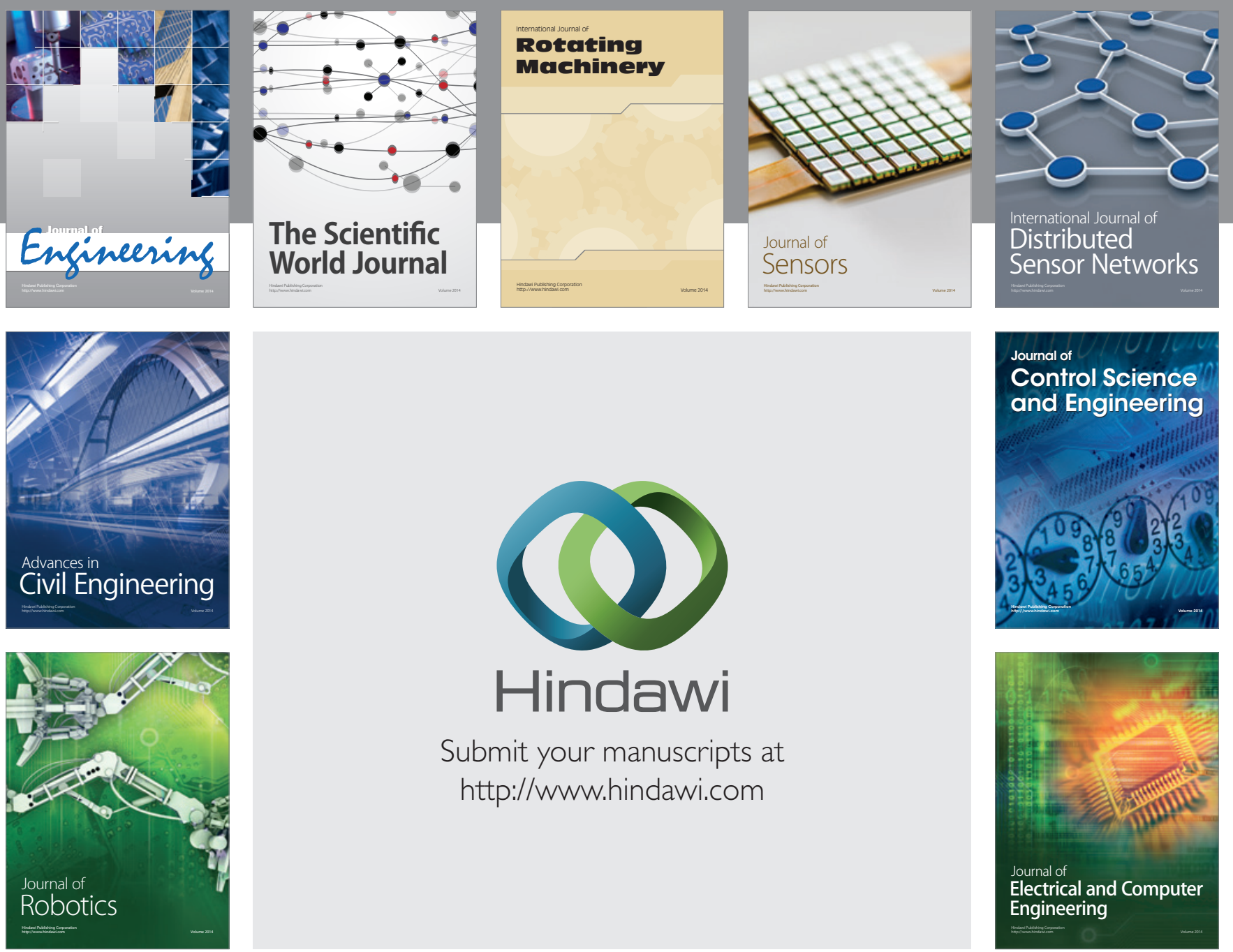

Submit your manuscripts at

http://www.hindawi.com
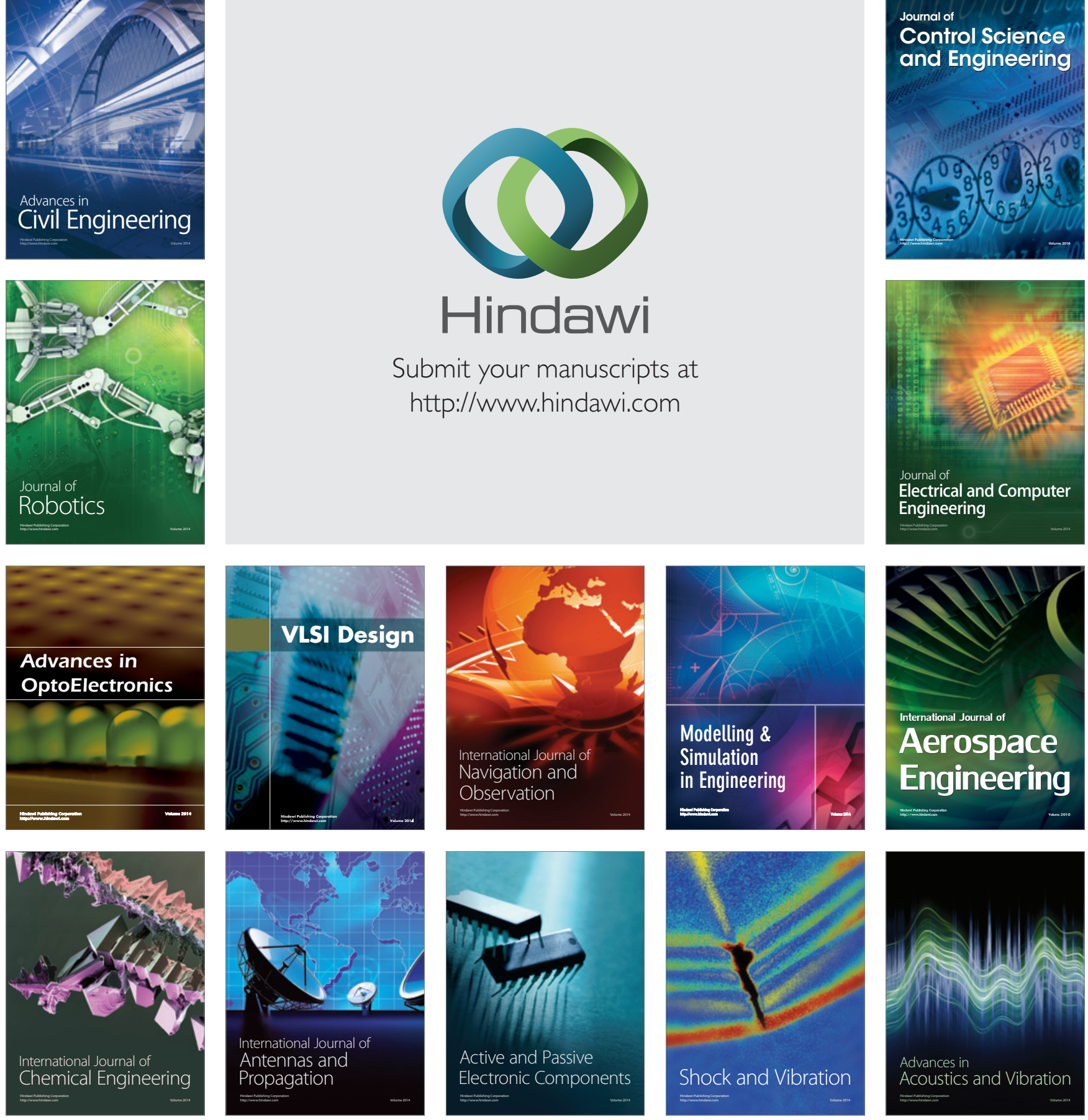